\title{
Kinetics and nucleation dynamics in ion-seeded atomic clusters
}

\author{
M. G. Rozman, ${ }^{1}$ M. Bredice $\odot,{ }^{1,}{ }^{*}$ J. Smucker $\odot,{ }^{1}$ H. R. Sadeghpour, ${ }^{2}$ D. Vrinceanu $\odot,{ }^{3}$ R. Côté, ${ }^{4}$ and V. Kharchenko ${ }^{1,2}$ \\ ${ }^{1}$ Department of Physics, University of Connecticut, Storrs, Connecticut 06269, USA \\ ${ }^{2}$ ITAMP, Center for Astrophysics | Harvard \& Smithsonian, Cambridge, Massachusetts 02138, USA \\ ${ }^{3}$ Department of Physics, Texas Southern University, Houston, Texas 77004, USA \\ ${ }^{4}$ Department of Physics, University of Massachusetts-Boston, Boston, Massachusetts 02125, USA
}

(Received 21 September 2021; revised 2 December 2021; accepted 21 January 2022; published 11 February 2022)

\begin{abstract}
The time-dependent kinetics of formation and evolution of nanosize atomic clusters is investigated and illustrated with the nucleation dynamics of ion-seed $\mathrm{Ar}_{n} \mathrm{H}^{+}$particles. The rates of growth and degradation of Ar-atomic shells around the seed ion are inferred from molecular dynamics (MD) simulations. Simulations of cluster formation have been performed with accurate quantum-mechanical binary interaction potentials. Both the nonequilibrium and equilibrium growth of $\mathrm{Ar}_{n} \mathrm{H}^{+}$are investigated at different temperature and densities of the atomic gas and seed ions. Formation of $\mathrm{Ar}_{n \leqslant 40}$ shells is the main mechanism which regulates the kinetics of nanocluster growth and the diffusive fluctuations of the cluster size distribution. The time evolution of the cluster intrinsic energy and cluster size distributions are analyzed at the nonthermal, quasiequilibrium, and thermal equilibrium stages of $\mathrm{Ar}_{n} \mathrm{H}^{+}$formation. We have determined the self-consistent model parameters for the temporal fluctuations of the cluster size and found coefficients of the diffusive growth mechanism describing the equilibrium distribution of nanoclusters. Nucleation of haze and nanodust particles in astrophysical and atmospheric ionized gases is discussed.
\end{abstract}

DOI: 10.1103/PhysRevA.105.022807

\section{INTRODUCTION}

The nature and characteristics of phase transitions in atomic and molecular systems strongly depend on the interparticle interactions. These binary interactions determine how rapidly new phases form and specifically regulate a nucleation of solid or liquid particles from gas and liquid phases. Classical nucleation theory (CNT) [1-4] was developed and successfully applied to the analysis of nucleation processes in macroscopic and submicron systems under conditions of thermal equilibrium. CNT predictions of the nucleation kinetics are essentially based on the Gibbs statistical rule and on stochastic (diffusive) formation of critical nucleation sizes $[1,2]$.

In contrast to CNT, theoretical modeling of nucleation and growth of nanoparticles under local nonequilibrium and nonhomogeneous conditions require precise knowledge of interatomic forces and a detailed description of relaxation processes. We found that nonhomogeneous nucleation of noble gas atoms, seeded by ions, is regulated via building up of ion-atom clusters from a sequence of stable atom shells. On the other hand, we observe large size fluctuations of nanoclusters under thermal equilibrium which resemble the diffusive

\footnotetext{
*mitchell.bredice@uconn.edu

Published by the American Physical Society under the terms of the Creative Commons Attribution 4.0 International license. Further distribution of this work must maintain attribution to the author(s) and the published article's title, journal citation, and DOI.
}

growth of critical clusters in CNT models. Nucleation of nanosize particles in ionized gases is an important process for a wide range of areas in physics, chemistry, astrophysics, and atmospheric sciences. Analysis of formation of nanosize haze and ice particles in the upper atmosphere of planets, satellites, exoplanets, and within debris disks is critically important for investigations of spectral properties of these atmospheres [5-10] and circumstellar disks [11-13]. Ions and charged nanodust particles are major agents stimulating haze and ice cloud formations in the Earth's upper atmosphere. The MD simulations, performed with accurate potentials of interparticle interaction, can successfully describe both nonequilibrium and equilibrium processes of nucleation of nanosize clusters. Previous investigations were focused on the nucleation [14,15], structural properties [16], and phase transitions of pure argon clusters [17,18]. Meanwhile, there is a significant amount of nanocluster research using supersonic beam experiments analyzing abundances of clusters with different numbers of atoms [19-29]. The theoretical analyses of cluster structure, $\mathrm{Ar}_{n} \mathrm{H}^{+}$[19,30-32] and small Lennard-Jones (LJ) clusters [33], have been concentrated on the explanation of different "magic numbers" obtained in different experiments. There is also significant work on small noble gas clusters focused on their fragmentation after ionization due to electron or proton impact [34-38]. Although nonequilibrium energy relaxation of excited clusters and following fragmentation involve the same binary potentials as cluster nucleation, the dynamics of these processes differ significantly. In our research, the formation and growth of nanosize $\mathrm{Ar}_{n} \mathrm{H}^{+}$clusters is initiated by ionization of $\mathrm{H}$ atoms in the $\mathrm{Ar}$ and $\mathrm{H}$ gas mixture and is simulated with the use of the Large-scale 
Atomic/Molecular Massively Parallel Simulator (LAMMPS) [39]. The nucleation of $\mathrm{Ar}_{n} \mathrm{H}^{+}$clusters have been investigated using the results of the MD simulations performed with quantum-mechanically calculated potentials and in the canonical: particle Number, Volume, Temperature (NVT) ensemble with the Nosé-Hoover thermostat [39]. The time-dependent kinetics of the nucleation and growth of critical $\mathrm{Ar}_{n} \mathrm{H}^{+}$clusters and their approach to thermal equilibrium have been inferred from the results of our MD simulations. A role of ion seed particles in a phase transition has been analyzed in detail employing data of our simulations performed with different parameters of the Ar bath gas and densities of $\mathrm{H}^{+}$ions.

\section{SHELL STRUCTURE, ENERGY, AND STABILITY OF NANOSIZE CLUSTERS}

The accurate binary potentials of the Ar-Ar and $\mathrm{Ar}-\mathrm{H}^{+}$ interactions have already been used in our investigations of nucleation and growth of $\mathrm{Ar}_{n} \mathrm{H}_{m}^{+}$solid or liquid particles, where large size clusters include many protons [40]. We briefly provide information on the major characteristics of these potentials.

\section{A. Binary interaction potentials}

The comprehensive description of the binary interaction potentials used in our simulations is given in [40]. To summarize the details, the Ar-Ar binary interaction is a LJ 6-12 short-range van der Waals potential with a well depth of $0.012 \mathrm{eV}$ and an equilibrium atomic separation of $3.75 \AA$. The $\mathrm{Ar}-\mathrm{H}^{+}$interaction at its deepest is more than $4 \mathrm{eV}$, and it asymptotically leads to a $r^{-4}$ polarization potential. This interaction is much stronger than the $\mathrm{Ar}-\mathrm{Ar}$ interaction due to the ion Coulomb field polarizing the neutral atom. Thus, Ar- $\mathrm{H}^{+}$potential creates the bulk of the interparticle attraction in our simulations, while the Ar-Ar interaction does not substantially contribute to any attraction forces in our simulations of $\mathrm{Ar}_{n} \mathrm{H}^{+}$cluster growth with $n \leqslant 40$. On the other hand, the short-range Ar-Ar repulsion together with $\mathrm{Ar}-\mathrm{H}^{+}$potential controls parameters of the Ar shells. The ion-ion interaction is Debye shielded and is modeled as a Yukawa-type interaction in LAMMPS. A sketch of the potentials is given in Fig. 1. All potentials used in our modeling do not include explicit three-body or higher many-body contributions.

\section{B. Geometrical structures and cluster energies: Emergence of primitive symmetry structures}

Nucleation of nanosize clusters has been studied in detail for neutral noble gases [41]. Neutral atoms at low temperatures form cluster shells minimizing the cluster energy. Larger cluster shells with increasing numbers of atoms can be transformed to regular crystal structures, if the reservoir temperature is below the melting point. Stability of nanocluster shells and their geometrical parameters is sensitive to characteristics of interatomic potentials [41]. Nucleation of atoms seeded by ions or charged nanoparticles differs significantly from cluster formation in homogeneous neutral gases. The strong attraction force between a seed ion and Ar atoms dominates the dynamics during the initial stages of $\mathrm{Ar}_{n} \mathrm{H}^{+}$

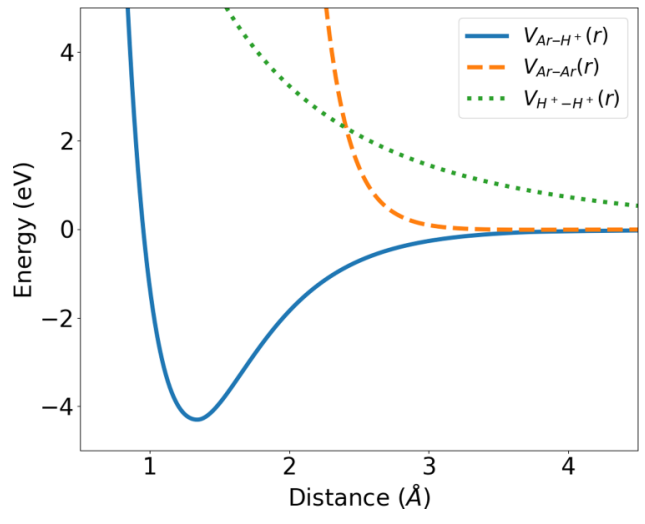

FIG. 1. Potential energy curves for the MD simulations. The screened $\mathrm{H}^{+}-\mathrm{H}^{+}$interaction is Debye shielded and modeled as a Yukawa-type potential, due to the electron and proton fields. The Ar-Ar interaction is modeled by a LJ potential and describes the short-range vdW interaction between $\mathrm{Ar}$ atoms. The $\mathrm{Ar}-\mathrm{H}^{+}$interaction asymptotically leads to a $r^{-4}$ polarization potential and is the longest range attraction in our simulation. The repulsion and attraction energies of the $\mathrm{Ar}-\mathrm{Ar}$ and $\mathrm{Ar}-\mathrm{H}^{+}$interactions are approximately equalized at $\sim 2.5 \AA$.

formation. This ionic force creates tighter confinement of the $\mathrm{Ar}_{n} \mathrm{H}^{+}$cluster with larger binding energies for Ar atoms in close proximity to the $\mathrm{H}^{+}$. The distance between $\mathrm{Ar}$ atoms and ion, i.e., the shell radius $\bar{r}$, is the same for all atoms belonging to the same shell, and the shell radius $\bar{r}$ practically does not depend on the number of atoms in the shell. The number of $\operatorname{Ar}$ atoms $n_{s}$ in the specific shell is restricted due to conditions minimizing a cluster potential energy. The maximal value $n_{s}$ occurs for the closed shells with $n_{s}=4,6, \ldots$. The specific geometrical configurations, tetrahedral and octahedral, are the primitive symmetry configurations around the central seed ion that minimize the potential energy $U(n)$ of clusters with closed shells. The primitive symmetry of the first Ar atomic shell, tetrahedral, disagrees with the structure found in the literature [19,30-32], which has the innermost layer of the cluster being a linear $\mathrm{Ar}-\mathrm{H}^{+}$-Ar cluster. Nevertheless, we expect that simulations of the cluster growth and cluster size distributions cannot be affected strongly by the semiclassical approximation used in our MD model. This is because in the both semiclassical and quantum-mechanical models, the cluster growth of $\mathrm{Ar}_{n-1} \mathrm{H}^{+}+\mathrm{Ar} \rightarrow \mathrm{Ar}_{n} \mathrm{H}^{+}$occurs via captures of Ar atoms into highly excited configuration states with a following relaxation of the cluster intrinsic energy to the ground states. The energies, $U(n)$, which correspond to the minimal total energies at zero temperature, are given in Table I together with an indication of the symmetry of the cluster configurations (shells), $\mathrm{Ar}-\mathrm{H}^{+}$interatomic distances $\bar{r}$, and shortest Ar-Ar distances within each shell that provide these minima.

The Ar atoms from the same shell have identical parameters, such as binding energy or an averaged distance from the cluster ion $\bar{r}$. The minimal energies $u(n)$ of Ar atoms in the cluster shell, $u(n)=U(n)-U(n-1)=d U / d n$, were calculated for small $\mathrm{Ar}_{n<25} \mathrm{H}^{+}$clusters and results are shown in Fig. 2 as a function of $n$. The detachment energy required to remove an $\mathrm{Ar}$ atom from a $\mathrm{Ar}_{n} \mathrm{H}^{+}$cluster is $|u(n)|$. The values 
TABLE I. The minimal potential energy $U(n)$, average radii $\bar{r}$, and shortest Ar-Ar (within each shell) are shown for the cluster structure with closed cluster shells $1 \mathrm{~T}^{4}$ with the number of Ar atoms $n_{S}=4,1 \mathrm{~T}^{4} 2 \mathrm{~T}^{4}$ with $n_{S}=8$, and $1 \mathrm{~T}^{4} 2 \mathrm{~T}^{4} 3 \mathrm{O}^{6}$ with $n_{S}=14$. The shell symmetries are contained in the notation, $\mathrm{T}=$ tetrahedral, $\mathrm{O}=$ octahedral. The shell radius $\bar{r}$ practically does not depend on the number of atoms in the specific shell and has the same values for closed and open shells. Details of geometrical structure of the small nanosize clusters and their minimal energies can be found at [42].

\begin{tabular}{lcccr}
\hline \hline Cluster & Symbol & $\bar{r}(\AA)$ & $r_{\text {Ar-Ar }}(\AA)$ & $U(n)(\mathrm{eV})$ \\
\hline $\mathrm{Ar}_{4} \mathrm{H}^{+}$ & $1 \mathrm{~T}^{4}$ & 1.69 & 2.76 & -10.2333 \\
$\mathrm{Ar}_{8} \mathrm{H}^{+}$ & $1 \mathrm{~T}^{4} 2 \mathrm{~T}^{4}$ & $1.69,3.57$ & $2.76,5.83$ & -10.6406 \\
$\mathrm{Ar}_{14} \mathrm{H}^{+}$ & $1 \mathrm{~T}^{4} 2 \mathrm{~T}^{4} 3 \mathrm{O}^{6}$ & $1.69,3.58,4.29$ & $2.76,5.84,6.07$ & -11.1789 \\
\hline \hline
\end{tabular}

of $u(n)$ take into account the mean potential field created by all cluster Ar atoms and the $\mathrm{H}^{+}$. The single-particle energy $u(n)$ plays the role of the chemical potential at $T=0$. The value of $u(n)$ at $\mathrm{n} \leqslant 4$ rises sharply with $n$ and then plateaus at $n \geqslant 4$. The four Ar atoms form the stable tetrahedral shell of atoms closest to the seed ion. The energy required to remove any of the Ar atoms from the $\mathrm{Ar}_{4} \mathrm{H}^{+}$shell at $T=0$ is $u(n=4) \simeq$ $0.64 \mathrm{eV}$, which corresponds to the temperature $\sim 7300 \mathrm{~K}$. This energy is significantly higher than thermal energies considered here, and the $\mathrm{Ar}_{4} \mathrm{H}^{+}$clusters are therefore stable in our simulations. A detachment of an Ar atom from the next tetrahedral shell $\left(2 \mathrm{~T}^{4}\right.$ in Table I) requires $0.1 \mathrm{eV} \sim 800 \mathrm{~K}$. This shell should be stable at low temperatures $(T \sim 90 \mathrm{~K})$ but can be depopulated under thermal equilibrium at $T \sim 200 \mathrm{~K}$ at sufficiently dilute Ar densities [43]. The ion field removes the diffusion barrier [2,3] of nucleation for small clusters and accelerates their nucleation. The charge-induced growth of small clusters occurs via the capture of free Ar atoms into cluster shells. The growth of $\mathrm{Ar}_{n} \mathrm{H}^{+}$particles is restricted by thermal detachment of Ar atoms from cluster shells or by formation of new phases; for example, the stable $\mathrm{Ar}_{n} \mathrm{H}_{m}^{+}$large clusters or

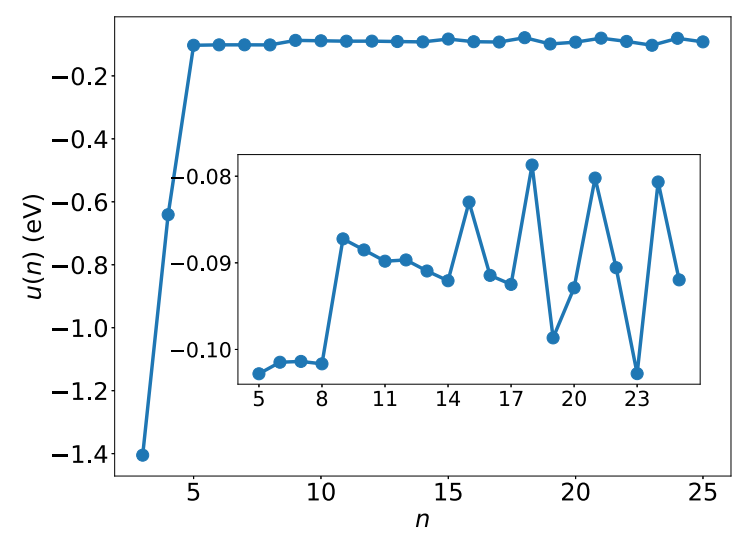

FIG. 2. The energies $u(n)$ of an Ar particle in the cluster shells, $u(n)=U(n)-U(n-1)=d U / d n$, as a function of the number of Ar atoms $n$ in the cluster. The minimal energy required at $T=0$ to remove an $\mathrm{Ar}$ atom from the $\mathrm{Ar}_{n} \mathrm{H}^{+}$cluster is $|u(n)|$. The sharp increase of the $u(n)$ function at $n \leqslant 4$ corresponds to construction of the deepest $1 \mathrm{~T}$ Ar shell. The plateau at $n \geqslant 5$ shows that shell energies $u(n)$ of larger clusters are weakly sensitive to the value of cluster size $n$. The energy $|u(n)|$ is the maximal energy which can be transferred to the thermostat in $\mathrm{Ar}_{n-1} \mathrm{H}^{+}+\mathrm{Ar} \rightarrow \mathrm{Ar}_{n} \mathrm{H}^{+}$transitions. The irregular oscillations of $u(n)$, shown in the insert, reflect energy $n$ alternation of Ar atoms in the open shells. The value of these oscillations is reduced at $n \gg 1$. crystals at low temperatures [40]. In our current simulations, the stable cluster shells with large number of Ar atoms were not observed at low densities or high temperatures. This is because the rate of evaporation of cluster-bound Ar atoms increases under these conditions significantly with respect to the shell growth rate causing depopulation of outer shells.

\section{METHODS AND RESULTS OF MD SIMULATIONS OF $\mathrm{Ar}_{n} \mathrm{H}^{+}$CLUSTER FORMATION}

Each MD simulation begins by randomly generating coordinates for the atoms and ions; the atoms and ions are separated by a minimum of $3 \AA$. The simulations are run at temperatures, $90<T<600 \mathrm{~K}$, with a variable number of ions from 0 to 200, and contain a fixed number of Ar atoms (1000) in the simulation box. Ar atom velocities are initialized with the Maxwell-Boltzmann distribution appropriate to the selected temperature, using the LAMMPS built-in "create velocity" function, but the initial velocity of the $\mathrm{H}^{+}$ions is assigned to be zero. To achieve different densities of Ar atoms and $\mathrm{H}^{+}$ions the size of the simulation box is adjusted, with the majority of the simulations performed at the Ar atom density of $10^{20} \mathrm{~cm}^{-3}$. This density is chosen to represent a dense gas and allows for a faster convergence of the cluster growth. All simulations are run using the canonical (NVT) ensembles with the Nose-Hoover thermostat function contained in LAMMPS. The time step for our simulations was $1 \mathrm{fs}$, and the thermostat temperature damping timescale was 100 fs to avoid sharp changes of the kinetic energies of atomic particles.

\section{A. Cluster definition}

The method to extract the clusters from the results of simulations is done in two stages, by first a geometrical selection of groups of close atoms, clusters, using the Density Based Spatial Clustering of Applications with Noise (DBSCAN) algorithm [44], with its implementation in the Julia programming language through the CLUSTERING.JL package, and next the computation of the cluster intrinsic energies to verify that all atoms in these clusters are bound.

Stage 1: The DBSCAN algorithm searches for neighbors around every atom in the simulation, and if another atom is within a defined cutoff distance $d$, then it is selected to be a part of a possible geometric $\mathrm{Ar}_{n} \mathrm{H}^{+}$cluster. In this first step we search only for clusters that include a single ion and use a cutoff distance of $d=4 \AA$. This cutoff was chosen from the energy minimization calculations, to ensure that the outer 
Ar shells with $n<40$ can be detected. The geometric cutoff selection could include "false" selections, e.g., a group of atoms and ions which are accidentally close to each other in one specific snapshot of the simulation, but not the next. Such groups of unbound particles are dissolved in a short time. These candidates for the $\mathrm{Ar}_{n} \mathrm{H}^{+}$clusters can be analyzed and rejected on the second stage of the cluster-verification process.

Stage 2: We implement another step to our definition, that the cluster total energy in the center of mass (CM) frame must be negative, since this would indicate a truly bound system. The Stage 2 verification yields values of atomic binding energies; this allows the comparison to the minimal cluster energies obtained in minimization calculations for each cluster size (Fig. 2). The irregular $n$-variations of $u(n)$ reflect the reconstruction of cluster shells with the addition of new atoms. The $n$-variations of the optimal configuration of cluster shells have been established in classical and quantum calculations of atomic binding energies [19,30,31]. These variations should vanish at " $n \rightarrow \infty$ " when $u(n)$ provides the minimal potential energy of atomic particle on the surface of a macroscopic $\mathrm{Ar}$ crystal at $T=0$. The real total potential energies for clusters obtained in our simulations are slightly larger than the minimum potential energy. The intrinsic cluster energy obtained in simulations is typically a few percent higher than the minimal potential energy. This excess of the intrinsic energy arises from the vibration of Ar atoms in the cluster (the kinetic energy of thermal motion), since our minimal potential energy is calculated at $T=0$.

\section{B. Simulations of the nucleation kinetics}

We have performed a set of MD simulations of the $\mathrm{Ar}_{n} \mathrm{H}^{+}$ cluster growth at different temperatures and concentrations of $\mathrm{Ar}$ atoms and charged particles $\mathrm{H}^{+}$. Nascent $\mathrm{H}^{+}$ions produced at $t=0$ in the thermal Ar gas quickly become centers of cluster nucleation. The formation of first atomic shells around charge centers is accompanied by a local release of significant energy. This initial stage of the nucleation process can be described as a nonthermal phase of the cluster formation. The large fraction of released energy is transferred to light particles, the protons, and later distributed between atomic particles and absorbed by the LAMMPS thermostat. Growth of cluster shells leads to diminishing binding energies of $\mathrm{Ar}$ atoms in $\mathrm{Ar}_{n} \mathrm{H}^{+}$clusters. Thus, the energy release is reduced with cluster growth and the entire system (Ar gas and $\operatorname{Ar}_{n} \mathrm{H}^{+}$clusters) approaches thermal equilibrium.

The MD simulations provide near complete information about spatial and velocity distributions of all atomic particles in the free $\mathrm{Ar}$ gas and in $\mathrm{Ar}_{n} \mathrm{H}^{+}$clusters. Every time interval $\delta t=20 \mathrm{ps}$, data are dumped for the entire simulation. This value of $\delta t$ allows to observe different phase space configurations at considered densities and temperatures of atomic particles. Analysis and calculations of physical and geometrical parameters are performed using these 20 ps snapshots.

\section{Cluster formation and velocity distributions of $\mathrm{Ar}$ and $\mathrm{H}^{+}$particles}

The initial stages of cluster growth occur in a nonequilibrium regime, lasting for the first $\sim 1-5 \mathrm{~ns}$. The duration of this nonequilibrium stage depends on the initial conditions, the Ar gas parameters, and density of $\mathrm{H}^{+}$ions. This is apparent from the analysis of $\mathrm{Ar}$ and $\mathrm{H}^{+}$velocity distributions. We see that after the first few steps (each step is 20 ps long), the $\mathrm{Ar}$ and $\mathrm{H}^{+}$velocity distributions are non-Maxwellian. In each case the majority of the atoms and ions have small velocities, but with long high-velocity tails. The energy source for the hot particles in high-velocity tails is the formation of small clusters: capture of free $\mathrm{Ar}$ atoms into an open $\mathrm{Ar}_{n} \mathrm{H}^{+}$cluster shells with $n \leqslant 8$ is an exothermic process with a large energy release, as shown in Fig. 2. Energy release decreases with growth of outer cluster shells and becomes comparable with values of typical thermal energy $k T$.

As the simulation progresses, the peak of the velocity distribution shifts until the distribution becomes nearly Maxwellian. Although an insignificant tail of high velocities persists in the $v$ distributions for the entire duration of the simulations, the bulk of the atoms and ions remain in the thermalized Maxwellian-type distribution. The time evolution of $\mathrm{Ar}$ and $\mathrm{H}^{+}$velocity distribution functions are show in Figs. 3(a) and 3(b). The argon atoms appear cold at 200 ps due to the large energy losses: Ar atoms transfer their kinetic energy to the initially "frozen" light particles (the ions) at the beginning of the simulation. This specific aspect of the velocity relaxation does not influence formation of the first $\mathrm{Ar}_{n} \mathrm{H}^{+}$shell, because atomic binding energies in the cluster shell are roughly two orders of magnitude larger than thermal energies.

\section{Nonequilibrium and equilibrium stages of cluster nucleation and growth \\ 1. Stages of $\mathrm{Ar}_{n} \mathrm{H}^{+}$cluster formation}

Analysis of the results of MD simulations shows at least three different phases in a formation of nanosize $\mathrm{Ar}_{n} \mathrm{H}^{+}$clusters. In our cluster growth simulations, all small clusters with $n \leqslant 40$ have distinct shell structures minimising their potential energies.

The growth of the cluster size $n$ corresponds to a consequent filling of unoccupied $\mathrm{Ar}_{n} \mathrm{H}^{+}$shells described in Sec. II. In Figs. 4(a) and 4(b), we show an example of the $\mathrm{Ar}_{n} \mathrm{H}^{+}$ growth and degradation for the time $t \leqslant 100 \mathrm{~ns}$ after $200 \mathrm{H}$ atoms have been ionized and mixed with the Ar gas. The numbers of clusters $N_{c}(n, t)$ with the specified number of $\mathrm{Ar}$ atoms $n$ are shown as functions of the time $t$ with the regular snapshot interval of $20 \mathrm{ps}$. Simulations have been carried at the Ar atom density of $10^{20} \mathrm{~cm}^{-3}$ and the temperature $T=$ 200 K. In Figs. 4(a) and 4(b) we can identify three distinct phases of nanoparticle nucleation and growth.

The first phase of nucleation is nonequilibrium nucleation $(t \lesssim 2 n s)$. During this stage, Ar atoms are "captured" into the deepest shells. Protons create a strong potential field, and the process of capturing Ar atoms into closest cluster shells releases energies comparable with $u(n)$. Thermal energy fluctuations cannot detach Ar atoms from the first deeply bound shell (Fig. 2). The stage is an irreversible nonequilibrium process of the formation of the inner atomic shells of $\mathrm{Ar}_{n} \mathrm{H}^{+}$. The reduction of small cluster population $N_{c}(n, t)$ for $n=1-3$ shown in Fig. 4(a) is explained by a capture of free Ar atoms into the closed cluster shells $1 \mathrm{~T}^{4}$. Outer cluster shells are 


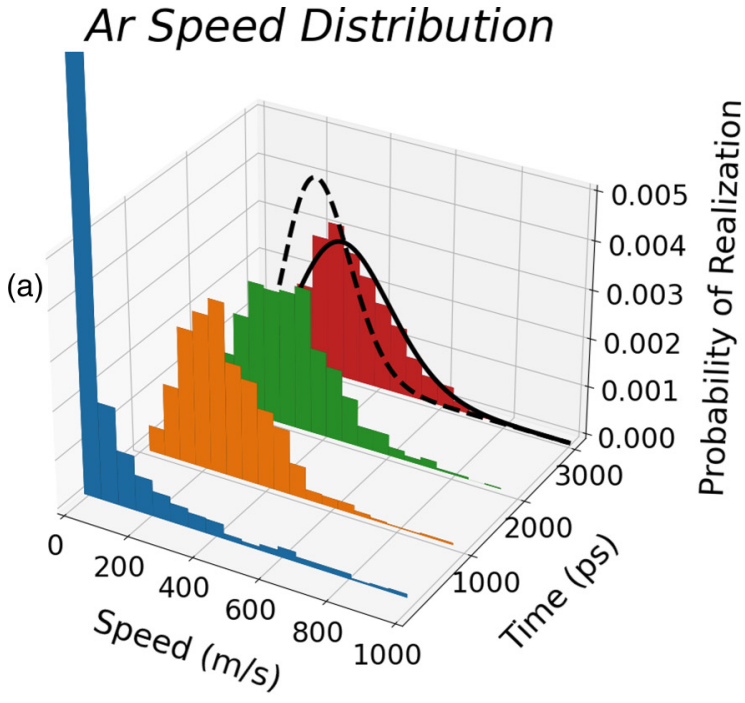

$H^{+}$Speed Distribution

(b)

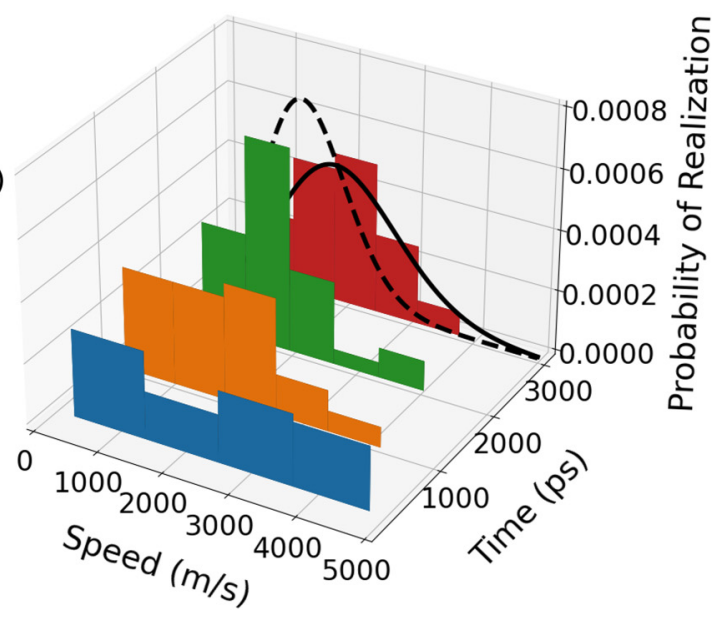

FIG. 3. The time evolution of $\mathrm{Ar}$ gas velocity distributions (a) and $\mathrm{H}^{+}$ions (b) during growth of $\mathrm{Ar}_{n} \mathrm{H}^{+}$clusters at $200 \mathrm{~K}$. The Ar atom density is $10^{20} \mathrm{~cm}^{-3}$ with $1000 \mathrm{Ar}$ atoms and $60 \mathrm{H}^{+}$ions in the simulation box. The small fraction of $\mathrm{Ar}$ atoms and $\mathrm{H}^{+}$ions in the high-velocity tail are omitted. The distributions are normalized to the unity. The theoretical Maxwell-Boltzmann velocity distributions are shown for $100 \mathrm{~K}$ (dashed line), and $200 \mathrm{~K}$ (solid line) together with results of simulations at $3 \mathrm{~ns}$. These figures illustrate the timescale required for the relaxation of velocity distribution functions.

formed at around $t \sim 0.1-1 \mathrm{~ns}$. The cluster nucleation dynamics can be illustrated by the time-dependent abundance of $\mathrm{Ar}_{n} \mathrm{H}^{+}(1 \leqslant n \leqslant 10)$ clusters shown in Figs. 4(a) and 4(b). At large $t$, nonsignificant populations of larger clusters with $n \geqslant 5$ reflect an efficient thermal evaporation of Ar atoms from outer cluster shells at $T=200 \mathrm{~K}$. $\mathrm{Ar}_{5} \mathrm{H}^{+}$clusters are more abundant than other states of $2 T^{n}$ shells due to the $n$-diffusive behavior of the relatively stable $\mathrm{Ar}_{4} \mathrm{H}^{+}$clusters. Details of the diffusive regime will be described in Sec. IV.

The second stage is quasiequilibrium growth $(2 \mathrm{~ns} \lesssim t \lesssim$ $60 \mathrm{~ns}$ ). This stage in the interval between $2 \mathrm{~ns}<t<60 \mathrm{~ns}$ is characterized by distinct fluctuations of the cluster size
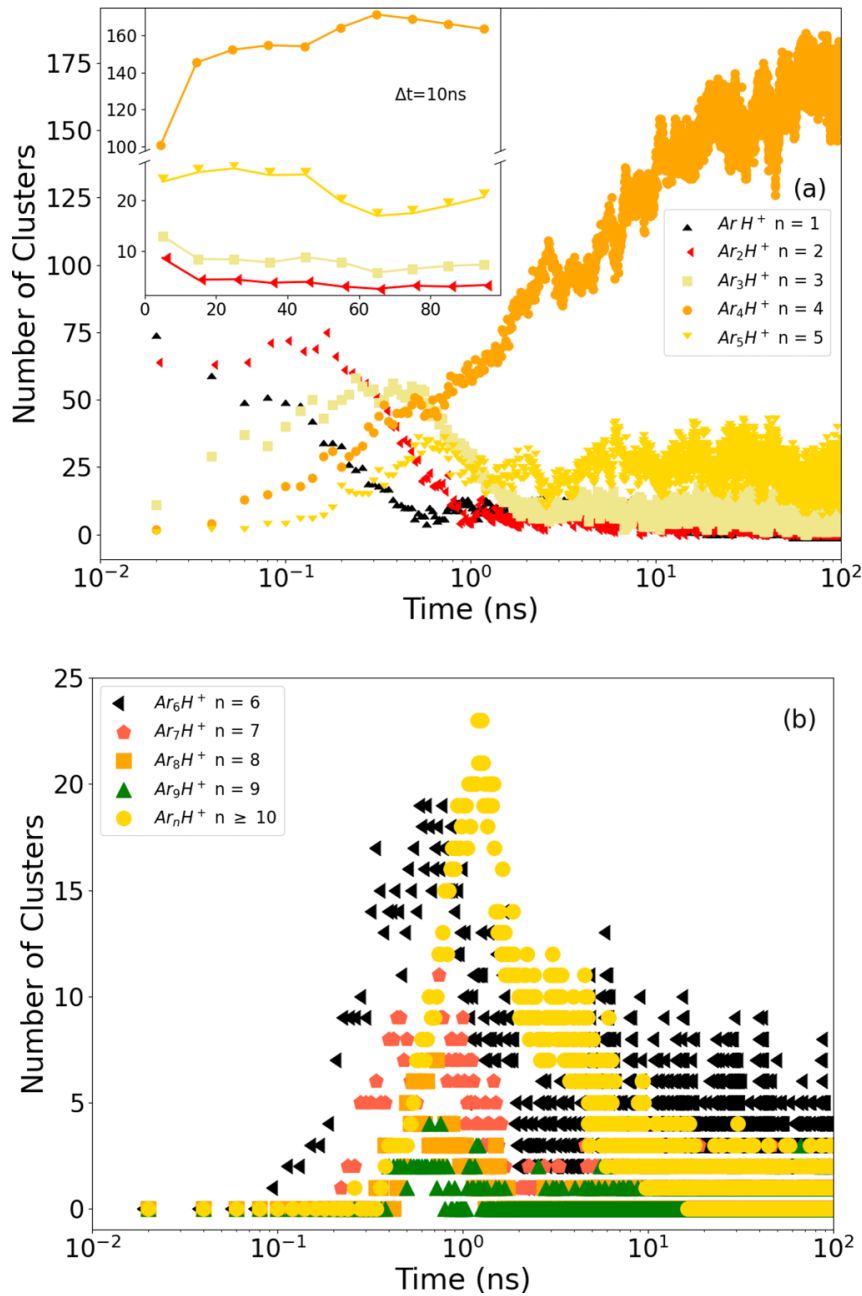

FIG. 4. The abundances $N_{c}(n, t)$ of $\mathrm{Ar}_{n} \mathrm{H}^{+}$clusters with different number $(n)$ of $\operatorname{Ar}$ atoms $(n \leqslant 5$ in (a), $6 \leqslant n \leqslant 9$ and the cumulative abundance of $n \geqslant 10$ in (b)] as functions of time $t$. Clusters are formed by an ensemble of $200 \mathrm{H}^{+}$ions embedded into the equilibrium $\mathrm{Ar}$ gas at the temperature $T=200 \mathrm{~K}$ and the atom density of $10^{20} \mathrm{~cm}^{-3}$. H atoms have been ionized at the time $t=0$. The inset axes in (a) show the abundances $N_{c}(n, t)$ of $\mathrm{Ar}_{n} \mathrm{H}^{+}(2 \leqslant n \leqslant 5)$ averaged over the interval $\Delta t=10 \mathrm{~ns}$.

shown in Fig. 4(a). During this quasiequilibrium stage of the nucleation kinetics, the tetrahedral $\mathrm{Ar}_{4} \mathrm{H}^{+}$clusters became the most abundant particles. The fluctuations of the cluster abundances relate to captures and losses of Ar atoms into specific cluster shells. Averaging of these fluctuations over statistically significant time intervals with values between 10 and $20 \mathrm{~ns}$ yields the smooth functions describing the average number of clusters $\bar{N}_{c}(n, t)$ with $n \mathrm{Ar}$ atoms. The number of clusters depicted in Fig. 4(a) have been averaged over statistically significant interval $\Delta t=10 \mathrm{~ns}$ and obtained results for $\bar{N}_{c}(n, t)$ are shown in the inset axes of Fig. 4(a). The steady growth of $\bar{N}_{c}(n=4, t)$ of tetrahedral clusters during this second stage can be considered as a quasiequilibrium nucleation and growth of a new phase.

The third stage is thermal equilibrium growth and size evolution $(t \gtrsim 60 n s)$. The growth of $\bar{N}_{c}(n=4, t)$ is stopped in this stage at $t \geqslant 60 \mathrm{~ns}$, when the system (free Ar atoms + 


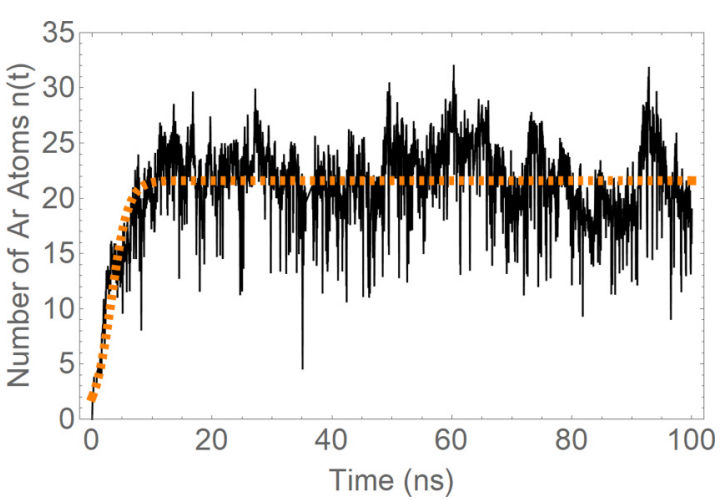

FIG. 5. MD simulations of number of $\mathrm{Ar}$ atoms $n(t)$ in a single $\mathrm{Ar}_{n(t)} \mathrm{H}^{+}$cluster. The simulation time is $t=100 \mathrm{~ns}$, the Ar gas temperature is $T=90 \mathrm{~K}$, and the number of Ar atoms in the simulation box $N=10^{3}$. The dashed line is the theoretical curve for an average cluster size $\bar{n}(t)$ as a function of time. The time delay parameter $t_{d}=3.2 \mathrm{~ns}$, and the scaling relaxation time is $t_{T}=1.4 \mathrm{~ns}$.

$\mathrm{Ar}_{n} \mathrm{H}^{+}$clusters) has reached the steady-state regime in cluster nucleation and evaporation, i.e., the system has relaxed to the state of full thermal equilibrium between different phases [Fig. 4(a)]. The averaged number of $\mathrm{Ar}_{n} \mathrm{H}^{+}$remain constant for the entire duration of the third stage $60 \mathrm{~ns}<t<100 \mathrm{~ns}$, and typical fluctuations of the $n$ number of Ar atoms bound by $\mathrm{H}^{+}$ions are described by thermal fluctuations. The time boundaries for these stages, in Figs. 4(a) and 4(b), are given as approximates, since the actual time boundaries between stages depend on $\mathrm{Ar}$ and $\mathrm{H}^{+}$densities and temperatures used in each specific simulation.

\section{Dynamics of the independent growth of $\mathrm{Ar}_{n} \mathrm{H}^{+}$clusters}

Under conditions of the independent growth of $\mathrm{Ar}_{n} \mathrm{H}^{+}$ clusters, the averaged time-dependent characteristics of a single cluster describe parameters of the ensemble of independent protons. This ergodic statement is not valid for interacting clusters, when consolidation of $\mathrm{Ar}_{n} \mathrm{H}^{+}$clusters leads to formation of a new phase like large $\mathrm{Ar}_{n} \mathrm{H}_{m}^{+}$nanocrystals or liquid droplets [40]. The nucleation and growth of independent clusters have been simulated for a single $\mathrm{H}^{+}$ ion at different temperatures and at the constant number of Ar atoms $N=10^{3}$ in the simulation box. Nucleation of the smallest $\mathrm{Ar}_{n} \mathrm{H}^{+}$clusters and consequent growth of Ar shells have been studied up to $t=100 \mathrm{~ns}$. In Fig. 5 the actual time evolution of the cluster size $n(t)$ is shown for a single $\operatorname{Ar}_{n(t)} \mathrm{H}^{+}$ cluster at $T=90 \mathrm{~K}$. The dashed red curve shows the theoretical data on the cluster growth based on a simplified two-state approximation [45]. In this model, the shell growth and cluster size oscillations are described by transitions between two states: the state of free $\mathrm{Ar}$ atoms in the simulation box of volume $V$ and the bound state of Ar atoms in the outer shell of the cluster of size $\bar{n}(t)$. We consider only the binding energies $\epsilon(n)$ of Ar atoms in the outer cluster shells and substitute instead $n(t)$ its values averaged over fluctuations: $n \rightarrow \bar{n}(t)$. The $\epsilon(n)$ energy in a specific shell depends on a mean field created by $\mathrm{H}^{+}$and all Ar atoms attached to the ion. The self-consistent energy $u(n)$ is an essential part of the binding energy $\epsilon(n)$, although $\epsilon(n)$ may include contributions of different shell configurations mixed by thermal fluctuations. We assume, for simplicity, that cluster growth occurs in subsequent captures and detachments of a single Ar atom $\bar{n}(t) \rightarrow \bar{n}(t) \pm 1$ and the number of Ar atoms in the gas is large $N \gg \bar{n}(t)$. For the two-state model, each Ar atom can occupy the outer shell of the $\operatorname{Ar}_{\bar{n}(t)} \mathrm{H}^{+}$cluster or stays in the free gas state. The partition function $Z$ of an ensemble of Ar atoms can be written as

$$
Z=\frac{z_{\mathrm{Ar}}^{N}}{N !}, \quad z_{\mathrm{Ar}}=V / \lambda_{T}^{3}+g(\bar{n}(t)) \exp \left[-\frac{\epsilon(\bar{n}(t))}{k T}\right],
$$

where $z_{\mathrm{Ar}}$ is the partition function for a $\mathrm{Ar}$ atom under condition of the Maxwell-Boltzamnn statistics, and $\lambda_{T}=$ $\sqrt{2 \pi \hbar^{2} / m k T}$ is the thermal de Broglie wave of Ar atoms. The first term in the expression for $z_{\text {Ar }}$ corresponds to the occupied number of states in the classical gas with the fixed temperature and particle density $[N \gg \bar{n}(t)]$, and the second one corresponds to the contribution of the outer cluster shell with the energy $\epsilon(\bar{n}(t))$ and statistical weight $g(\bar{n}(t))$. The values of $g(\bar{n}(t))$ are approximately proportional to the product of geometrical cluster volume $V_{c}(\bar{n}(t))$ and the thermal momentum space volume: $g(\bar{n}(t)) \sim V_{c}(\bar{n}(t)) / \lambda_{T}^{3}$. The presence of $N_{\mathrm{H}^{+}}$ independent $\mathrm{H}^{+}$ions in the simulation box could increase the cluster statistical weight by $N_{\mathrm{H}^{+}}$times.

The two-state approximation describes the populations of these states via the effective chemical potential $\mu_{\text {eff }}$, defined for Ar atoms in the outer cluster shell. The $\mu_{\text {eff }}$ value takes into account different statistical weights of the gas and cluster states [45]. Our simulations include the strong nonequilibrium initial condition: all deep cluster shells are empty at $t=0$. Under this condition, the value of $\mu_{\text {eff }}$, regulating population of the cluster shells should depend on time: $\mu_{\mathrm{eff}}(t)=$ $\mu_{\text {eff }}(N, T, t)$. This effective chemical potential will asymptotically approach the equilibrium value $\mu(N, T)$ when the relaxation processes are accomplished. The population of cluster shells $\bar{n}(t)$ can be expressed as

$$
\bar{n}(t)=N \frac{\exp [-\Delta(t, T)]}{1+\exp [-\Delta(t, T)]} \simeq n_{c} \frac{\exp \left[\frac{\left(t-t_{d}\right)}{t_{T}}\right]}{1+\exp \left[\frac{\left(t-t_{d}\right)}{t_{T}}\right]},
$$

where $n_{c}=\bar{n}(t \rightarrow \infty)$ is the average number of Ar atoms in the cluster under condition of thermal equilibrium, and $\Delta(t, T)=\frac{\epsilon(\bar{n}(t))-\mu_{\text {eff }}(t)}{k T}$. The parameter $t_{T}$ represents the scaling time of cluster growth during the non-Maxwellian and quasiequilibrium stages of nucleation. The scaling time-shift parameter $t_{d}$ takes into account a time delay in formation of first tetrahedral shells and formally describes a motion of $\mu_{\mathrm{eff}}(t)$ towards the upper cluster shells with the atomic binding energy $\epsilon(n)$. The full derivation of the time delay in the formation of $\mathrm{ArH}^{+}, \mathrm{Ar}_{2} \mathrm{H}^{+}$, and $\mathrm{Ar}_{3} \mathrm{H}^{+}$molecules needs to include an accurate analysis of few-body collisions, which are out of the scope of the simple two-state model. Thus, the simplified time dependence of $\bar{n}(t)$ from Eq. (2) cannot provide accurate initial conditions at $t=0$, but it describes well an evolution of $\bar{n}(t)$ for the entire time of simulations.

The reduction of the absolute value $\left|\mu_{\text {eff }}(t)\right|$ with time leads to the population of cluster shells with smaller binding energies $|\epsilon(n)|$ and thus stimulates an increase of the average cluster size $\bar{n}(t)$. At $t \rightarrow \infty$ the asymptotic value of $\mu_{\text {eff }}(t)$ matches to the equilibrium chemical potential $\mu(N, T)$ : $\mu_{\mathrm{eff}}(t) \rightarrow \mu(N, T)$, and $\bar{n}(t \rightarrow \infty) \rightarrow n_{c}$. In Fig. 5 the value 

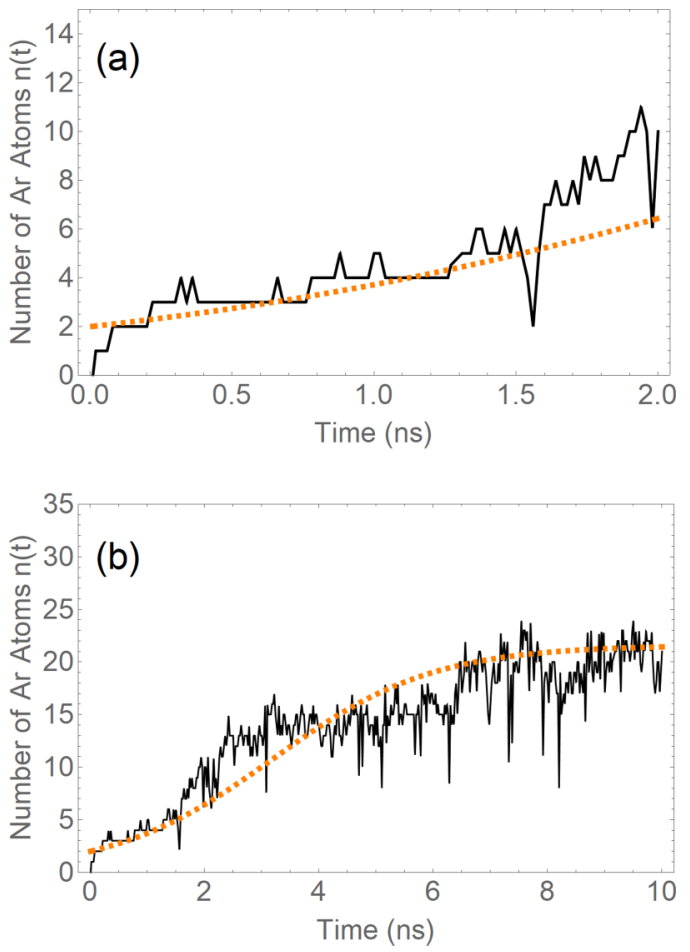

FIG. 6. The number of Ar atoms $n(t)$ in the single $H^{+}$ion cluster as a function of the time in the nonequilibrium stage of nucleation: (a) Formation of the deep shells in $\mathrm{Ar}_{n} \mathrm{H}^{+}$clusters for the time interval $t=0-2$ ns after creation of the $\mathrm{H}^{+}$ion in the argon bath gas. (b) The intermediate stage of $\mathrm{Ar}_{n} \mathrm{H}^{+}$growth and development of the thermal fluctuations of $n(t)$ inside the time interval $0<t<10 \mathrm{~ns}$. The gas temperature and density are $T=90 \mathrm{~K}$ and $\mathrm{n}_{g}=10^{20} \mathrm{~cm}^{-3}$, respectively. Theoretical curve is computed using thermodynamic formula for the two-state approximation given by Eq. (2) with the kinetic parameters $t_{d} \simeq 3.2 \mathrm{~ns}, t_{T}=1.4 \mathrm{~ns}$, and $n_{c}=21.6$.

of $\mu_{\mathrm{eff}}(t)$ has been shifted up to energies $\epsilon(n)$ of $\mathrm{Ar}_{n} \mathrm{H}^{+}$ clusters with $n \sim 10-14$ for the time interval of few units of scaling time $t_{d}$, i.e., approximately for $3 t_{d} \sim 10 \mathrm{~ns}$. This reflect the process of the time-dependent population of the three deepest cluster shells of Ar atoms. From Eq. (2) we can conclude, that at $t=t_{d}$ an average size $\bar{n}(t)$ has to be about $50 \%$ of its thermal equilibrium value $n_{c}=21.6$ at $T=90 \mathrm{~K}$. The values of kinetics parameters, $t_{d} \simeq 3.2 \mathrm{~ns}$ and $t_{T}=1.4 \mathrm{~ns}$, are inferred from the data depicted in Fig. 5. To illustrate the efficiency of the two-state model, we show in Figs. 6(a) and 6(b) the initial stages of cluster growth for two time intervals $0<t \lesssim 2$ ns and $0<t \lesssim 10$ ns. The nonequilibrium stage $(0<t \lesssim 2 \mathrm{~ns})$ in Fig. 6(a) does not indicate any detachments of Ar atoms from the first, deepest cluster shell. This nonequilibrium stage of growth cannot yield notable fluctuations of the cluster size $n(t)$ because the binding energies of Ar atoms in small clusters $(n \leqslant 8)$ are significantly larger than the thermal energy at $T=90 \mathrm{~K}$ and thermal fluctuations cannot remove Ar atoms from these shells. Rare changes of $n(t)$ in Fig. 6(a) are considered as attempts to increase the cluster size. Time of formation of the first two tetrahedral shells is estimated as 1-1.5 ns. The scaling value of thermalization time $t_{T}=1.4 \mathrm{~ns}$ is in good agreement with our evaluation of the velocity relaxation time of Ar atoms. The $t_{T}$ value can be identified in Figs. 4(a), 4(b), 5, 6(a), and 6(b) as the onset of the fast and strong up and down fluctuations of the cluster size $n(t)$ with a simultaneous steady increase of the mean cluster size $\bar{n}(t)$. The quasiequilibrium stage of cluster growth begins after $1.5-2 \mathrm{~ns}$. The mean cluster size $\bar{n}(t)$ increases slowly until it shows a saturation at 10-15 ns since the beginning of the nucleation process, as is depicted in Figs. 5 and 6(b).

\section{EQUILIBRIUM SIZE DISTRIBUTION OF INDEPENDENT Ar $\mathrm{H}^{+}$CLUSTERS AND CLUSTER FLUCTUATIONS}

The size distribution of solid or liquid nanoparticles is a fundamental characteristic required for analysis and modeling of many astrophysical and atmospheric phenomena. The size distribution of small clusters depends on interatomic interactions and the nucleation kinetics. The attachment and detachment of atoms or molecules create time-dependent fluctuations of the cluster size even under the thermal equilibrium condition. Detailed analysis of these fluctuations can provide information on the cluster size distribution in an ensemble of independent particles.

\section{A. Fluctuations of cluster size under the thermal equilibrium condition}

The "flat" behavior of $\bar{n}(t)$ at large time $t$ and intensive fluctuation regime are indicators of the thermal equilibrium between "cluster-bound" and free Ar atoms. The number of Ar atoms $n(t)$ in a $\mathrm{Ar}_{n} \mathrm{H}^{+}$cluster are shown in Fig. 7(a) for two clusters formed by two independent protons under the thermal equilibrium conditions. Two "trajectories" of MD simulations $n_{1}(t)$ and $n_{2}(t)$ for this independent clusters are shown as functions of time inside the time interval between 40 and $44 \mathrm{~ns}$. Cluster size fluctuations are characterized by different timescales and amplitudes. Averaging of $n(t)$ function over different time intervals shows separate typical frequency and amplitudes of cluster size fluctuations. For example, the results of averaging (filtering) of the cluster size fluctuations are shown in Fig. 7(b) for different Gaussian filtering intervals $2 \sigma=40 \mathrm{ps}, 0.2 \mathrm{~ns}$, and $1 \mathrm{~ns}$. Fast fluctuations correspond to the thermal attachment and detachment of Ar atoms from cluster shells. Slow fluctuations can be attributed to a longterm relaxation of thermodynamical parameters of the gas environment around clusters. The long-term fluctuations exist for all $\mathrm{Ar}_{n} \mathrm{H}^{+}$clusters and they have similar scale of typical fluctuations, but happen at different times and cluster locations.

The fluctuation pattern of the cluster size distribution $N_{c}(n, t)$ has also been found in simulations of cluster growth for an ensemble of $\mathrm{H}^{+}$ions embedded into Ar gas [Figs. 4(a) and 4(b)]. Different stages of nucleation processes shown in Figs. 4(a) and 4(b) for the ensemble of $200 \mathrm{H}^{+}$ions and thermal fluctuations of the cluster population can be investigated in detail using data of the cluster formation by individual $\mathrm{H}^{+}$ ions. Strong time fluctuations of $n(t)$ near the average equilibrium value illustrate the diffusive nature of cluster growth under the thermal equilibrium conditions. The scale of cluster fluctuations shown in Figs. 7(a) and 7(b) provides accurate 

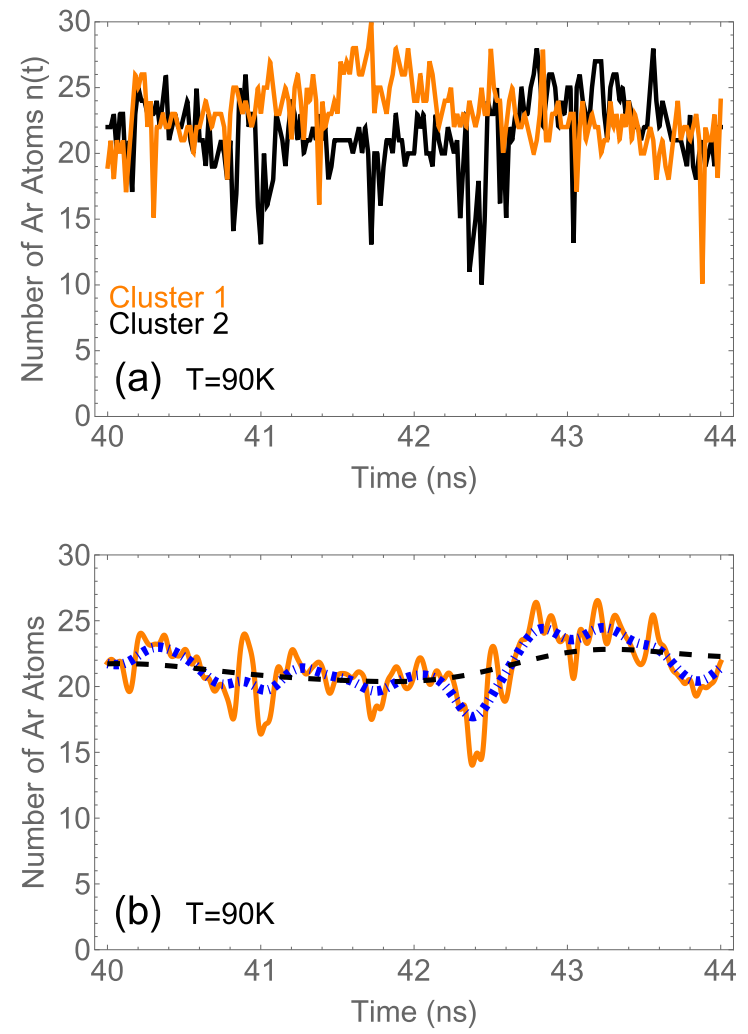

FIG. 7. (a) The number of Ar atoms $n_{1}(t)$ and $n_{2}(t)$ in two independent clusters, 1 and 2, are shown as functions of the time $t$ under the thermal equilibrium conditions inside the time interval $t=$ 40-44 ns. The gas temperature and density are $T=90 \mathrm{~K}$ and $\mathrm{n}_{g}=$ $10^{20} \mathrm{~cm}^{-3}$, respectively. (b) The timescale filtering of fluctuations are shown with the smoothed curves shown for cluster 2 from (a). The averaging time for the Gaussian filters are $2 \sigma=40 \mathrm{ps}, 0.2 \mathrm{~ns}$, and $1 \mathrm{~ns}$ for solid, dot-dashed, and dashed curves, respectively.

information on the stationary equilibrium distribution of $\mathrm{Ar}_{n} \mathrm{H}^{+}$cluster sizes at different temperatures.

\section{B. Equilibrium size distribution of $\mathrm{Ar}_{n} \mathrm{H}^{+}$clusters}

Parameters of the time evolution of the average cluster size $\bar{n}(t)$ and time-dependent fluctuations, $n(t)-\bar{n}(t)$, around the average value yield unique information on the cluster size distribution during the quasiequilibrium and equilibrium stages of the cluster growth. Fluctuations can be considered as the bases for the diffusion process in the parametric $\{n\}$ space of the $\mathrm{Ar}_{n} \mathrm{H}^{+}$clusters [1-4]. Exchange of Ar atoms between cluster shells and free Ar gas during the quasiequilibrium or equilibrium stages of nucleation is an example of a random walk in the space of cluster size $n$.

The diffusion characteristics of the $\mathrm{Ar}_{n} \mathrm{H}^{+}$growth and the stationary cluster size distributions $P(n, T)$ have been inferred from the results of MD simulations at different temperatures and ion concentrations. The empirical probabilities to detect $\mathrm{Ar}_{n} \mathrm{H}^{+}$cluster with $n$ Ar atoms during specified time interval $\tau$ is given by the normalized probability distribution function (PDF) $P(n, t, \tau, T)$. The empirical probability $P(n, t, \tau, T)=$ $N(n, t, \tau, T) / N(t, \tau, T)$ is defined as a ratio of the number of $N(n, t, \tau, T)$ realization of $\mathrm{Ar}_{n} \mathrm{H}^{+}$clusters with $n$ atoms

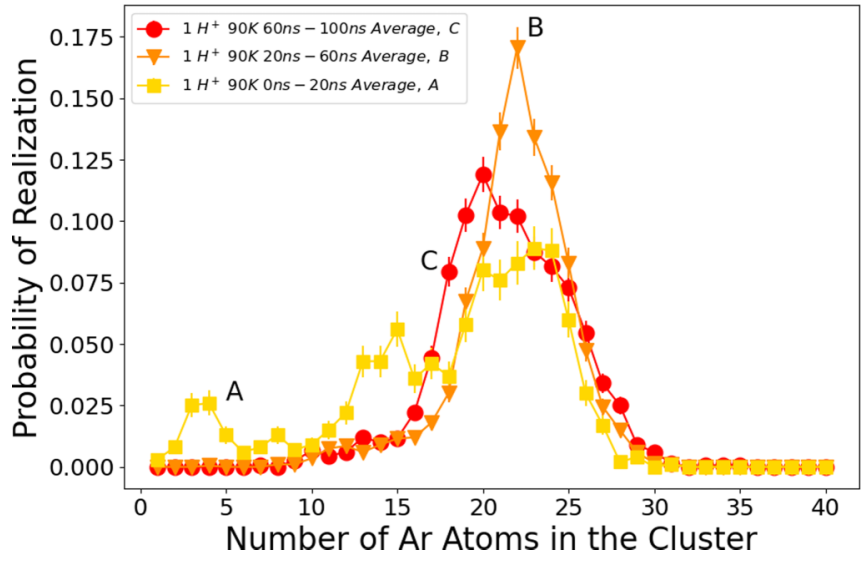

FIG. 8. The empirical probability $P(n, t, \tau, T)$ to find the $\mathrm{Ar}_{n} \mathrm{H}^{+}$ clusters with $n$ Ar atoms in the cluster shells. The total simulation time $t=100 \mathrm{~ns}$ for a single $\mathrm{H}^{+}$ion at $T=90 \mathrm{~K}$. The curves A, $\mathrm{B}$, and $\mathrm{C}$ are shown at different times: curve $\mathrm{A}$, the earlier stage of cluster formation $0 \leqslant t \leqslant 20 \mathrm{~ns}$; curve $\mathrm{B}$, just after thermalization $20 \leqslant t \leqslant 60 \mathrm{~ns}$; and curve $\mathrm{C}, 60 \leqslant t \leqslant 100 \mathrm{~ns}$ at the end of the MD simulation process. The averaging interval for the later distributions is $\tau=40 \mathrm{~ns}$, and for the early stage A is $20 \mathrm{~ns}$. The $0-20 \mathrm{~ns}$ time interval includes essentially nonequilibrium stage of the cluster nucleation and this causes a visible fraction of small clusters in the empirical probability given by curve $\mathrm{A}$.

for the time interval $\tau$ to the total number of realization $N(t, \tau, T)=\sum_{n} N(n, t, \tau, T)$. The time interval $\tau$ has been selected to be high enough for an accurate statistical evaluation of $n$ distributions. The defined PDF does not depend on the time, under condition of the thermal equilibrium and describes stationary thermal probabilities $P(n, T)$.

We have computed $P(n, t, \tau, T)$ at different time $t$ to investigate the kinetics of the independent cluster growth under quasiequilibrium and equilibrium conditions. Results are shown by curves $\mathrm{A}, \mathrm{B}$, and $\mathrm{C}$ in Fig. 8. The error bars in Fig. 8 indicate a standard deviation of the MD simulation results. Cluster size distributions at different times $t$ reflect different phases of the cluster shell formation. A long tail of small clusters with $n \leqslant 16$ as is shown in Fig. 8 (curve A), is formed during earlier stages of cluster growth under nonequilibrium conditions, and this is clearly reflected in $P(n, t, \tau=$ $20 \mathrm{~ns}, T)$ for $t \leqslant 20$. More detailed information about the cluster $n$ distributions at short time $t \leqslant 20$ ns requires significantly shorter intervals $\tau$ due to the fast nonthermal growth of clusters (Fig. 5) and so an increasing number of independent simulating trajectories $\{n(t)\}$.

The quasiequilibrium and equilibrium stages of the cluster formation arise after $t \geqslant 20 \mathrm{~ns}$ (B and C curves in Fig. 8). They show broad distributions of the cluster sizes $n$ between 15 and 30 Ar atoms. These probabilities are controlled by the distribution of the cluster Ar-detachment energies $u(n)$, which have deeper local minima near $n \sim 19-23$ as is shown in Fig. 2. The low temperature $T=90 \mathrm{~K}$ allows $\mathrm{Ar}$ atoms to occupy outer cluster shells. These shells intensively exchange by Ar atoms with the thermal Ar gas creating large $n$ fluctuations (Fig. 5) with the standard deviation $\sigma(n, T) \simeq$ $4 \mathrm{Ar}$ atoms. The value of $\sigma(n, t)$, inferred from the analysis of time-dependent fluctuations at the thermal equilibrium stage, 


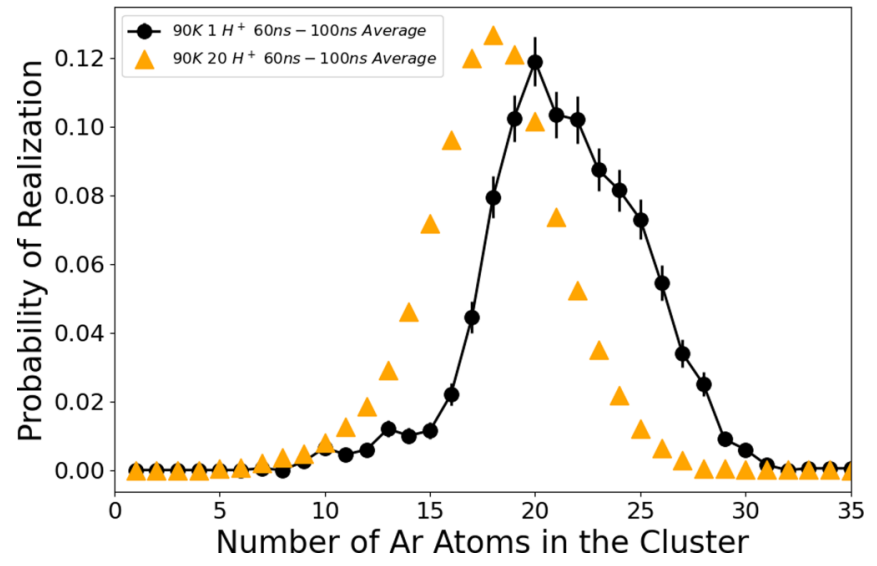

FIG. 9. The probability distribution function $P\left(n, N_{\mathrm{H}^{+}}\right)$to find $\mathrm{Ar}_{n} \mathrm{H}^{+}$clusters with $n$ Ar atoms in the cluster shells as a function

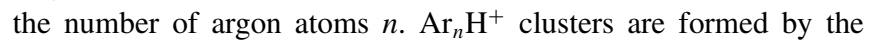
initial ensemble of $\mathrm{N}_{\mathrm{H}^{+}}$ions. The total simulation time is $t=100 \mathrm{~ns}$. The gas temperature $T=90 \mathrm{~K}$, with either $\mathrm{N}_{\mathrm{H}^{+}}=1$ (circles) or $\mathrm{N}_{\mathrm{H}^{+}}=20$ (triangles) $\mathrm{H}^{+}$ions, all at Ar density $10^{20} \mathrm{~cm}^{-3}$. Data are averaged over the time interval 60-100 ns; error bars for the $20 \mathrm{H}^{+}$ ion curve are on the order of the marker size. The introduction of more ions leads to a reduction of the mean cluster size compare with the independent proton growth.

allows to compute the theoretical value of the full width at half maximum (FWHM) of the thermal distribution: $\mathrm{FWHM} \simeq$ $2.34 \sigma(n, t) \simeq 9.3$. This matches well to the simple estimation of the $\mathrm{FWHM}_{C}$ value of the thermal distribution $P(n, T)$ (curve $\mathrm{C}$ in Fig. 8): $\mathrm{FWHM}_{C} \simeq 10$, if the $n$ distribution of the $\mathrm{C}$ curve is approximated by a Gaussian. The peak of $P(n, t, \tau, T)=P(n, T)$ around the minimal values of $u(n)$ at the region $n \sim 19-23$ and strong $n$ fluctuations are mostly formed at $t>20 \mathrm{~ns}$.

\section{KINETICS OF THE CLUSTER FORMATION FOR ENSEMBLE OF $\mathrm{H}^{+}$IONS}

The kinetics of cluster nucleation and their thermal equilibrium parameters can be modified by the mutual influence of $\mathrm{Ar}_{n} \mathrm{H}^{+}$in the cluster formation process. At high concentration of $\mathrm{H}^{+}$ions, the capture of free Ar atoms by different clusters becomes a competitive process. Additional complexity of nucleation arises at low gas temperature, when strong correlations between $\mathrm{Ar}_{n} \mathrm{H}^{+}$clusters lead to their aggregation to a new phase, the large-scale $\mathrm{Ar}_{n} \mathrm{H}_{m}^{+}$droplet or nanocrystal [40]. This transition may occur via several paths, such as consolidation of strongly bound $\mathrm{Ar}_{4} \mathrm{H}^{+}$clusters or as a coalescence process of absorption of small and medium clusters by larger nanoparticles [1]. We have simulated the cluster nucleation using different initial ensembles of $\mathrm{H}^{+}$ions, $\mathrm{N}_{\mathrm{H}^{+}}$from 1 to $200 \mathrm{H}^{+}$ions at the temperatures $T=90 \mathrm{~K}$ and $200 \mathrm{~K}$.

To clarify the influence of an increasing $\mathrm{H}^{+}$density on the nucleation process, we have performed simulations of the $\mathrm{Ar}_{n} \mathrm{H}^{+}$nucleation at $N_{\mathrm{H}^{+}}=1$ and $20 \mathrm{H}^{+}$ions at the temperature $T=90 \mathrm{~K}$ and the constant number of Ar atoms $N_{\mathrm{Ar}}=10^{3}$ in the simulation box. Results are shown in Fig. 9. The significant increase of the number of $\mathrm{H}^{+}$ions (triangles in Fig. 9 for $N_{\mathrm{H}^{+}}=20$ ) leads to a reduction of the mean cluster

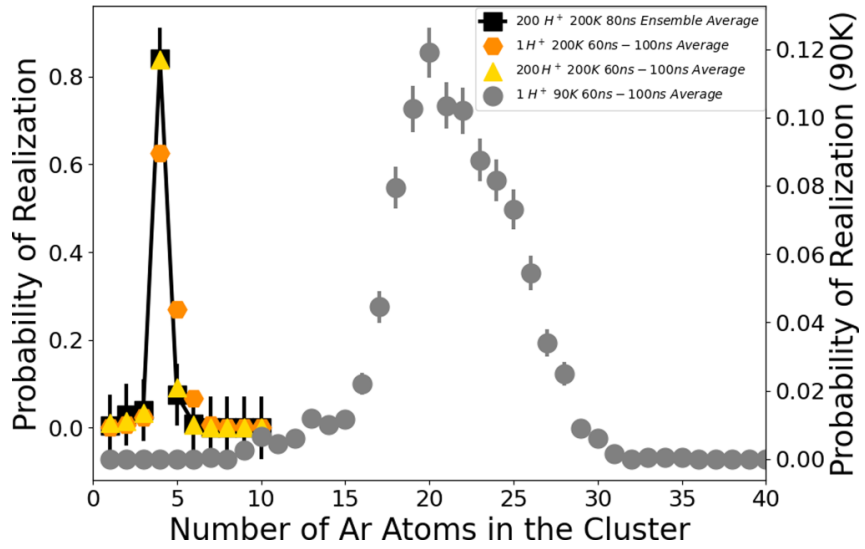

FIG. 10. The thermal equilibrium probability $P\left(n, N_{\mathrm{H}^{+}}, T\right)$ to detect the $\mathrm{Ar}_{n} \mathrm{H}^{+}$clusters with the $n$ Ar atoms for the ensembles of $\mathrm{N}_{\mathrm{H}^{+}}=1$ and $200 \mathrm{H}^{+}$ions. The results of the independent $\mathrm{H}^{+}$ion growth are show for $T=200 \mathrm{~K}$ (hexagons, left vertical axis) and $T=90 \mathrm{~K}$ (circles, right vertical axis). The cluster size distribution $P\left(n, N_{\mathrm{H}^{+}}=200, T=200 \mathrm{~K}\right)$ for the averaged value over the ensemble of $200 \mathrm{H}^{+}$ions at the single snapshot at $t=80 \mathrm{~ns}$ are shown (squares, left vertical axis) with corresponding error bars. The triangles (left vertical axis) show results of $P\left(n, N_{\mathrm{H}^{+}}=200, T=200 \mathrm{~K}\right)$ obtained with time and ensemble averaging. The error bars for this case is smaller than the marker size.

size compared with the independent proton growth (circles). At $T=90 \mathrm{~K}$, the $20 \mathrm{H}^{+}$ions have captured about $36 \%$ of free argon atoms. The density of the free Ar gas became around $64 \%$ of the initial gas density and that shifts the negative chemical potential of free Ar atoms down towards Ar cluster binding energies on the value $\Delta \mu_{\text {gas }}(T) \simeq \ln (0.64) \mathrm{kT} \simeq$ $-0.45 \mathrm{kT}$. Argon atoms from the largest size clusters are easily evaporated due to such shifts. In this simplified estimations we have neglected changes of the mean-field potential.

The cluster size distribution for the ensemble of $200 \mathrm{H}^{+}$ ions has been inferred from our MD simulations at the temperature $T=200 \mathrm{~K}$ and results are shown in Fig. 10 by the triangles obtained with time and ensemble averaging. The squares with corresponding error bars shows the same data for $T=200 \mathrm{~K}$ averaged only over the ensemble of $200 \mathrm{H}^{+}$ at the single snapshot of $t=80 \mathrm{~ns}$. This narrow size distribution is peaked around $\mathrm{Ar}_{4} \mathrm{H}^{+}$clusters. Clusters with large atomic shells cannot be observed at high temperatures because thermal fluctuations destroy these shells. For example, at the temperature $T=200 \mathrm{~K}$ only a small cluster with the typical size between two and seven Ar atoms in the shells can be stable. For comparisons between multiple-proton and single-proton nucleation, the equilibrium distribution function of the cluster size $P(n, T=200 \mathrm{~K})$ has been inferred from the single $\mathrm{H}^{+}$ion simulations of the independent cluster growth at $T=200 \mathrm{~K}$ and the results are shown in Fig. 10 with red down-triangles. The narrow $P(n, T=200 \mathrm{~K})$ distribution is sharply peaked at $n=4$ (the tetrahedral clusters) in contrast with the low-temperature $P(n, T=90 \mathrm{~K})$, which is favorable to a broad range of larger cluster size with $n \sim 15-30$. For comparison, the low-temperature $n$ distribution of independent clusters at $T=90 \mathrm{~K}$ is also shown in Fig. 10 by circles. 
The similarity of the high-temperature distributions for the single $\mathrm{H}^{+}$and $200 \mathrm{H}^{+}$ions at $T=200 \mathrm{~K}$ shows that although the cluster chemical potentials are sensitive to both the density of the $\mathrm{H}+$ ions and $\mathrm{Ar}$ atoms; the large value of the $\mathrm{Ar}$ binding energy of the $\mathrm{Ar}_{4} \mathrm{H}^{+}$cluster causes the cluster size distribution to be weakly sensitive to the proton density in the specific interval of temperature around $200 \mathrm{~K}$ considered in this article. Therefore cluster growth at chosen parameters can be seen mostly as a nucleation kinetics of independent small clusters. This claim is strongly supported by results of time-dependent kinetics of cluster nucleation in the ensemble of 200 seed $\mathrm{H}^{+}$ions previously shown in Figs. 4(a) and 4(b). The abundances ( $n$ distribution) of all small clusters with sizes $n \leqslant 10$ have reached their equilibrium values around 2 ns. However, the quasiequilibrium stage of nucleation (the orange circles and gold upside-down triangles [curve in Fig. 4(a) for the clusters $\mathrm{Ar}_{4} \mathrm{H}^{+}$and $\mathrm{Ar}_{5} \mathrm{H}^{+}$respectively] shows the steady growth of stable tetrahedral clusters [the circles in Fig. 4(a)]. The development of thermal fluctuations is clearly seen in Fig. 4(a) for $\mathrm{Ar}_{4} \mathrm{H}^{+}$and $\mathrm{Ar}_{5} \mathrm{H}^{+}$clusters. The antiphase of $\mathrm{Ar}_{4} \mathrm{H}^{+}$and $\mathrm{Ar}_{5} \mathrm{H}^{+}$fluctuations results in an effective single $\mathrm{Ar}$ atom exchange between the tetrahedral clusters and free Ar gas. Any capture of free $\mathrm{Ar}$ atoms by $\mathrm{Ar}_{4} \mathrm{H}^{+}$leads to formation of new $\mathrm{Ar}_{5} \mathrm{H}^{+}$clusters and to reduction of $\mathrm{Ar}_{4} \mathrm{H}^{+}$. The thermal equilibrium detachment of a single Ar atom from $\mathrm{Ar}_{5} \mathrm{H}^{+}$produces a new stable tetrahedral cluster. The thermal energy fluctuation cannot destroy the deepest shell even at $T=200 \mathrm{~K}$. The $\mathrm{Ar}_{4} \mathrm{H}^{+}$are most stable and abundant clusters for both the single- and multiple-proton nucleation process as is shown in Fig. 10.

\section{CONCLUSIONS}

Our analysis of MD simulations provide a consistent scenario for a nucleation phase initiated by ions in neutral gases. The growth of ion clusters occurs via the formation of atomic shells around the ion seed particle. MD simulations show evidence for three distinct stages of cluster nucleation: nonequilibrium, quasiequilibrium, and equilibrium stages. In the first nonequilibrium stage, the strong ion field removes the barriers to nucleation; thus, the probability to capture a gas atom into the deeply cluster-bound states (1T and $2 \mathrm{~T}$ ) is significantly higher than the probability for all detachment processes. This leads to the build up of inner shells during this stage. When a gas atom is captured into inner shells, a local release of high kinetic energies occurs. This causes the energy distributions of the atomic particles to become non-Maxwelllian. Once these energy distributions relax to a Maxwellian-like distribution; the system moves into the second quasiequilibrium stage. During this stage the equilibrium between the new phase (clusters) and the free gas has yet to be established. Thus, we see a steady growth of the cluster size and number of clusters in multiple-ion systems, up to the final thermal equilibrium stage. The notable feature of the quasiequilibrium stage is the onset and evolution of fast and strong fluctuations of the atom number. The size of the cluster increases with simultaneous decrease of binding energies in the outer cluster shells. This stimulates detachment processes and the exchange of atoms between the clusters and the free gas. Then system moves into the final thermal-equilibrium, stage, where the cluster size distribution reaches a steady state value. The density of gas and ions, temperature, and parameters of cluster shell structures regulate the cluster sizedistribution. The time-dependent fluctuations of the cluster size can be used to predict parameters of the steady-state distribution, specifically, the cluster size dispersion in an ensemble of growing clusters.

Nucleation of nanoparticles in astrophysical and planetary environments include more complicated physics of the cluster formation in molecular gases, such as $\mathrm{N}_{2}, \mathrm{CO}_{2}, \mathrm{H}_{2} \mathrm{O}, \mathrm{CH}_{4}$, and others; due to the internal molecular structure and more complicated intermolecular and intramolecular interactions. We intend to investigate kinetics of nanocluster formation in important astrophysical gases and apply theoretical parameters of nucleation for modeling observational data. Our results reported in this article will be useful in analysis of the cluster growth rates and size distributions of charge-seeded clusters as function of the gas parameters and molecular structures.

\section{ACKNOWLEDGMENTS}

The work of M.B., J.S., and R.C. on this project was supported via the UCLA Grant No. ONRBAA13-022. H.R.S. and V.K. acknowledge support from the NSF through a grant for ITAMP at Harvard University. D.V. is grateful for the support received from the National Science Foundation through Grants No. PHY-1831977 and No. HRD-1829184.
[1] E. M. Lifshitz and L. Pitaevskii, Physical Kinetics (IOP Publishing, Oxford, UK, 2010)

[2] Y. Zeldovich, Selected Works of Yakov Borisovich Zeldovich, Vol. 1 (Princeton University Press, Princeton, 1992).

[3] E. Cloet, in ASM Handbook, Fundamentals of Modeling for Metals Processing, edited by D. U. Furrer and S. L. Semiatin (ASM International, 2009), Vol. 22a, pp. 203-219.

[4] S. Karthika, T. K. Radhakrishnan, and P. Kalaichelvi, Cryst. Growth Des. 16, 6663 (2016).

[5] W. Grundy, T. Bertrand, R. Binzel, M. Buie, B. Buratti, A. Cheng, J. Cook, D. Cruikshank, S. Devins, C. Dalle Ore, A. Earle, K. Ennico, F. Forget, P. Gao, G. Gladstone, C. Howett,
D. Jennings, J. Kammer, T. Lauer, I. Linscott et al., Icarus 314, 232 (2018).

[6] A. Wellbrock, A. J. Coates, G. H. Jones, V. Vuitton, P. Lavvas, R. T. Desai, and J. H. Waite, Mon. Not. R. Astron. Soc. 490, 2254 (2019).

[7] P. Gao, D. P. Thorngren, E. K. H. Lee, J. J. Fortney, C. V. Morley, H. R. Wakeford, D. K. Powell, K. B. Stevenson, and X. Zhang, Nat. Astron. 4, 951 (2020).

[8] A. D. James, J. S. A. Brooke, T. P. Mangan, T. F. Whale, J. M. C. Plane, and B. J. Murray, Atmos. Chem. Phys. 18, 4519 (2018).

[9] A. V. Egorov, E. N. Brodskaya, and A. Laaksonen, Colloid J. 80, 484 (2018). 
[10] X. Yang and A. W. Castleman Jr., J. Geophys. Res.: Atmos. 96, 22573 (1991).

[11] A. V. Krivov, I. Mann, and N. A. Krivova, Astron. Astrophys. 362, 1127 (2000).

[12] A. V. Krivov, T. Löhne, and M. Sremcević, Astron. Astrophys. 455, 509 (2006).

[13] I. Mann, Philos. Trans. R. Soc. A 375, 20160254 (2017).

[14] K. Laasonen, S. Wonczak, R. Strey, and A. Laaksonen, J. Chem. Phys. 113, 9741 (2000).

[15] T. Kraska, J. Chem. Phys. 124, 054507 (2006).

[16] V. V. Hoang and T. Odagaki, Phys. Rev. B 77, 125434 (2008).

[17] R. S. Dumont, S. Jain, and A. G. Basile, J. Chem. Phys. 102, 4227 (1995).

[18] C. Rey, L. Gallego, M. Iñiguez, and J. Alonso, Phys. B: Condens. Matter 179, 273 (1992).

[19] M. Gatchell, P. Martini, L. Kranabetter, B. Rasul, and P. Scheier, Phys. Rev. A 98, 022519 (2018).

[20] H. Schöbel, P. Bartl, C. Leidlmair, S. Denifl, O. Echt, T. D. Märk, and P. Scheier, Eur. Phys. J. D 63, 209 (2011).

[21] T. Kurzthaler, B. Rasul, M. Kuhn, A. Lindinger, P. Scheier, and A. M. Ellis, J. Chem. Phys. 145, 064305 (2016).

[22] M. Kuhn, M. Renzler, J. Postler, S. Ralser, S. Spieler, M. Simpson, H. Linnartz, A. G. G. M. Tielens, J. Cami, A. Mauracher, Y. Wang, M. Alcamí, F. Martín, M. K. Beyer, R. Wester, A. Lindinger, and P. Scheier, Nat. Commun. 7, 13550 (2016).

[23] P. W. Stephens and J. G. King, Phys. Rev. Lett. 51, 1538 (1983).

[24] H. Buchenau, E. L. Knuth, J. Northby, J. P. Toennies, and C. Winkler, J. Chem. Phys. 92, 6875 (1990).

[25] I. A. Harris, R. S. Kidwell, and J. A. Northby, Phys. Rev. Lett. 53, 2390 (1984).

[26] I. Harris, K. Norman, R. Mulkern, and J. Northby, Chem. Phys. Lett. 130, 316 (1986).

[27] T. Märk and P. Scheier, Chem. Phys. Lett. 137, 245 (1987).

[28] M. Lezius, P. Scheier, A. Stamatovic, and T. D. Märk, J. Chem. Phys. 91, 3240 (1989).
[29] W. Miehle, O. Kandler, T. Leisner, and O. Echt, J. Chem. Phys. 91, 5940 (1989).

[30] D. C. McDonald, D. T. Mauney, D. Leicht, J. H. Marks, J. A. Tan, J.-L. Kuo, and M. A. Duncan, J. Chem. Phys. 145, 231101 (2016).

[31] K. T. Giju, S. Roszak, and J. Leszczynski, J. Chem. Phys. 117, 4803 (2002).

[32] J. A. Tan and J.-L. Kuo, J. Phys. Chem. A 124, 7726 (2020).

[33] K. Vafayi and K. Esfarjani, J. Cluster Sci. 26, 473 (2015).

[34] I. Janeček, P. Naar, M. Stachoň, F. X. Gadea, and R. Kalus, Phys. Chem. Chem. Phys. 19, 2778 (2017).

[35] F. Calvo, D. Bonhommeau, and P. Parneix, Phys. Rev. Lett. 99, 083401 (2007).

[36] D. Bonhommeau, N. Halberstadt, and U. Buck, Int. Rev. Phys. Chem. 26, 353 (2007).

[37] D. Bonhommeau, N. Halberstadt, and A. Viel, J. Chem. Phys. 124, 184314 (2006).

[38] E. Surdutovich and A. V. Solov'yov, Eur. Phys. J. D 73, 241 (2019).

[39] A. P. Thompson, H. M. Aktulga, R. Berger, D. S. Bolintineanu, W. M. Brown, P. S. Crozier, P. J. in 't Veld, A. Kohlmeyer, S. G. Moore, T. D. Nguyen, R. Shan, M. J. Stevens, J. Tranchida, C. Trott, and S. J. Plimpton, Comp. Phys. Comm. 271, 108171 (2022)

[40] O. C. F. Brown, D. Vrinceanu, V. Kharchenko, and H. R. Sadeghpour, Mol. Phys. 118, e1767813 (2020).

[41] B. M. Smirnov and R. Berry, Phase Transitions of Simple Systems (Springer, Heidelberg, 2007).

[42] https://www.phys.uconn.edu/ $\sim$ kharchenko/clusters/.

[43] E. M. Lifshitz and L. Pitaevskii, Statistical Physics, Course of Theoretical Physics Vol. 5 (Elsevier, Oxford, UK, 2010).

[44] M. Ester, H.-P. Kriegel, J. Sander, and X. Xu, Proceedings of the Second International Conference on Knowledge Discovery and Data Mining (AAAI Press, 1996), pp. 226-231.

[45] D. A. R. Dalvit, J. Frastai, and I. D. Lawrie, Problems on Statistical Mechanics (Elsevier, Philadelphia, 1999). 